\title{
Autoacetylation regulates differentially the roles of ARD1 variants in tumorigenesis
}

\author{
JI HAE SEO ${ }^{1}$, JI-HYEON PARK ${ }^{1}$, EUN JI LEE ${ }^{1}$, TAM THUY LU VO ${ }^{1}$, HOON CHOI $^{1}$, JAE KYUNG JANG ${ }^{1}$, \\ HEE-JUN WEE ${ }^{1}$, BUM JU AHN ${ }^{1}$, JONG-HO CHA ${ }^{1}$, MIN WOOK SHIN ${ }^{1}$ and KYU-WON KIM ${ }^{1,2}$ \\ ${ }^{1}$ SNU-Harvard Neurovascular Protection Research Center, College of Pharmacy and \\ Research Institute of Pharmaceutical Sciences, ${ }^{2}$ Department of Molecular Medicine and \\ Biopharmaceutical Sciences, Graduate School of Convergence Science and Technology, \\ and College of Medicine or College of Pharmacy, Seoul National University, \\ Seoul 151-742, Republic of Korea
}

Received May 6, 2014; Accepted June 27, 2014

DOI: 10.3892/ijo.2014.2708

\begin{abstract}
ARD1 is an acetyltransferase with several variants derived from alternative splicing. Among ARD1 variants, mouse $\mathrm{ARD}^{225}\left(\mathrm{mARD}^{225}\right)$, mouse $\mathrm{ARD}^{235}\left(\mathrm{mARD}^{235}\right)$, and human $\mathrm{ARD}^{235}$ (hARD1 ${ }^{235}$ ) have been the most extensively characterized and are known to have different biological functions. In the present study, we demonstrated that mARD1 ${ }^{225}$, mARD1 $1^{235}$, and hARD1 ${ }^{235}$ have conserved autoacetylation activities, and that they selectively regulate distinct roles of ARD1 variants in tumorigenesis. Using purified recombinants for ARD1 variants, we found that mARD1 ${ }^{225}, \mathrm{mARD}^{235}$, and hARD1 ${ }^{235}$ undergo similar autoacetylation with the target site conserved at the Lys136 residue. Moreover, functional investigations revealed that the role of $\mathrm{mARD}^{225}$ autoacetylation is completely distinguishable from that of $\mathrm{mARD} 1^{235}$ and hARD1 ${ }^{235}$. Under hypoxic conditions, mARD1 ${ }^{225}$ autoacetylation inhibited tumor angiogenesis by decreasing the stability of hypoxia-inducible factor- $1 \alpha$ (HIF- $1 \alpha)$. Autoacetylation stimulated the catalytic activity of $\mathrm{mARD} 1^{225}$ to acetylate Lys532 of the oxygen-dependent degradation (ODD) domain of HIF-1 $\alpha$, leading to the proteosomal degradation of HIF-1 $\alpha$. In contrast, autoacetylation of $\mathrm{mARD} 1^{235}$ and hARD1 ${ }^{235}$ contributed to cellular growth under normoxic conditions by increasing the expression of cyclin D1. Taken together, these data suggest that autoacetylation of ARD1 variants differentially regulates angiogenesis and cell proliferation in an isoform-specific manner.
\end{abstract}

Correspondence to: Dr Kyu-Won Kim, SNU-Harvard Neurovascular Protection Research Center, College of Pharmacy and Research Institute of Pharmaceutical Sciences, Seoul National University, Seoul 151-742, Republic of Korea

E-mail: qwonkim@snu.ac.kr

Key words: angiogenesis, ARD1 variant, autoacetylation, cell proliferation, cyclin D1, HIF- $1 \alpha$

\section{Introduction}

ARD1 was originally described as $\mathrm{N}$-acetyltransferase in Saccharomyces cerevisiae, where it is required for regulation of the cell cycle, mating, and sporulation (1). Subsequently, mammalian ARD1 was identified and is known to acetylate lysine residues of several proteins, including hypoxia-inducible factor- $1 \alpha$ (HIF-1 $\alpha), \beta$-catenin, myosin light chain kinase, the androgen receptor, and the tubulin complex (2-5). Several ARD1 variants produced from alternative splicing of mRNA have been identified in mouse and human cells $(6,7)$. Thus far, three mouse (mARD1198, mARD1 ${ }^{225}, \mathrm{mARD}^{235}$ ) and two human (hARD1131, hARD1 ${ }^{235}$ ) ARD1 variants were reported. Among these, mARD1 ${ }^{225}, \mathrm{mARD}^{235}$, and hARD1 ${ }^{235}$ have been most extensively studied and characterized.

mARD $1^{225}$ was first identified in mouse and found to negatively regulate angiogenesis. $\mathrm{mARD}^{225}$ acetylates HIF- $1 \alpha$ protein leading to its degradation via the ubiquitin-proteasome pathway (2). However, it was reported subsequently that other homologs of ARD1 (mARD1 ${ }^{235}$ and hARD1 ${ }^{235}$ ) could not alter HIF-1 $\alpha$ stability, suggesting different roles of ARD1 variants in the regulation of HIF-1 $\alpha(8-10)$. In contrast to the tumor suppression effects of mARD $1^{225}$, hARD1 ${ }^{235}$ is mainly known to contribute to tumorigenesis by enhancing cell proliferation. In many studies, the downregulation of hARD1 $1^{235}$ reduces cellular growth and induces cell cycle arrest $(3,11,12)$. Furthermore, increased expression of hARD1 ${ }^{235}$ is frequently observed in various human cancers, including breast, lung, and colorectal cancers (13-16). Thus, hARD $1^{235}$ is recognized as a critical oncogenic protein in cancer progression.

In a previous study, we reported that hARD $1^{235}$ has autoacetylation activity that is required for the stimulation of cancer growth by hARD1 ${ }^{235}$ (11). Based on this information, the present study was designed to compare the regulatory mechanisms of ARD1 variants and to investigate how they selectively regulate distinct functions of ARD1 variants that are involved in tumorigenesis. The results demonstrate that ARD1 variants have conserved autoacetylation activity that stimulates their catalytic activities. However, depending on 
the physiological conditions, this autoacetylation differentially regulates the biological functions of ARD1 variants in angiogenesis and cell proliferation in an isoform-specific manner.

\section{Materials and methods}

Reagents and antibodies. Anti-HIF-1 $\alpha$ antibody was purchased from BD Pharmingen. Anti-Myc and green fluorescent protein (GFP) antibodies were purchased from Santa Cruz Biotechnology. Anti-acetyl-lysine antibody was purchased from Cell Signaling. Anti-tubulin and Flag antibodies were purchased from Sigma-Aldrich. MG132 was purchased from Calbiochem.

Cell culture and hypoxic condition. HeLa cells and human umbilical vein endothelial cells (HUVECs) were grown in Dulbecco's modified Eagle's medium supplemented with $10 \%$ fetal bovine serum (FBS) and EBM-2 medium supplemented with growth factors (Lonza), respectively. Cells were maintained at $37^{\circ} \mathrm{C}$ in a humidified atmosphere containing $5 \% \mathrm{CO}_{2}$. Hypoxic conditions were created by incubating cells at $37^{\circ} \mathrm{C}$ in a chamber containing $5 \% \mathrm{CO}_{2}, 1 \% \mathrm{O}_{2}$, and the remainder $\mathrm{N}_{2}$.

Plasmid construction and transfection. To construct expression vectors for ARD1 variants, ARD1 cDNA was amplified by polymerase chain reaction (PCR) and subcloned into GFP- or Myc-tagged pCS2+ vectors for cell expression, and pGEX-4T for bacterial induction of the recombinant protein. Mutations in ARD1 were created using the Muta-Direct ${ }^{\mathrm{TM}}$ Site Directed Mutagenesis kit (Intron) according to the manufacturer's instructions. Cells were transfected with Lipofectamine (Life Technology) or Polyfect (Qiagen) according to the manufacturer's instructions.

Immunoblotting and immunoprecipitation. Cells were harvested and extracted in lysis buffer (10 mM HEPES at pH 7.9, $40 \mathrm{mM} \mathrm{NaCl}, 0.1 \mathrm{mM}$ ethylenediaminetetraacetic acid (EDTA), 5\% glycerol, $1 \mathrm{mM}$ dithiothreitol (DTT), and protease inhibitors). The concentrations of the protein extracts were measured with the BCA assay. For immunoprecipitations, relevant primary antibodies were added to $1 \mathrm{mg}$ of the protein extracts and incubated overnight at $4^{\circ} \mathrm{C}$. The immunoprecipitates and total cell lysates were resolved in sodium dodecyl-sulfate polyacrylamide gel electrophoresis gels and transferred onto nitrocellulose membranes (Amersham Pharmacia Bioscience). The membrane was probed with a primary antibody followed by a secondary antibody conjugated with horseradish peroxidase, and detected using an ECL system (Intron Biotechnology).

In vitro acetylation assay. Recombinants of GST-ARD1 variants were freshly prepared as described previously (11). These recombinants were incubated with or without His-tagged oxygen-dependent degradation (ODD) domain of HIF-1 $\alpha$ recombinants in the reaction mixture $(50 \mathrm{mM}$ Tris- $\mathrm{HCl}$ at pH 8.0,0.1 mM EDTA, 1 mM DTT, 10\% glycerol, and $10 \mathrm{mM}$ acetyl-CoA) at $37^{\circ} \mathrm{C}$.

Reverse transcription (RT)-PCR analysis. Total RNA was extracted using an RNA extraction kit (Invitrogen). cDNA was synthesized from $2 \mu \mathrm{g}$ of RNA using an oligo(dT) primer. Primers used for PCR were as follows: human $V E G F$, 5'-GAGAATTCGGCCTCCGAAACCATGAACTT TCTGCT-3' (forward) and 5'-GAGCATGCCCTCCTGCCC GGCTCACCGC-3' (reverse); ARD1, 5'-ATGAACATCCGC AATGCGAG-3' (forward) and 5'-CTCATATCATGGCT CGAGAGG-3' (reverse); cyclin D1, 5'-CTGGCCATGAA CTACCTGGA-3' (forward) and 5'-GTCACACTTGATCAC TCTGG-3' (reverse); GAPDH, 5'-ACCACAGTCCATGCCAT CAC-3' (forward) and 5'-TCCACCACCCTGTTGCTGTA-3' (reverse). The PCR amplification was carried out for 25 cycles with $A R D 1$, cyclin D1, and GAPDH, and for 30 cycles with $V E G F$.

Tube formation assay. For the tube formation assay, 24-well plates were coated with Matrigel (BD Biosciences) and allowed to polymerize at $37^{\circ} \mathrm{C}$ for $30 \mathrm{~min}$. HUVECs were seeded $\left(5 \times 10^{4}\right.$ cells per well) onto Matrigel with $500 \mu 1$ conditioned medium from HeLa cells. Tube formation was assessed after $4 \mathrm{~h}$ and quantified by determining the number of rings.

Cell proliferation assay. The cell growth rate was measured using a non-radioactive proliferation assay kit (Promega) according to the manufacturer's instructions. Briefly, cells were plated on 96-well plates and grown for 3 days. Substrate solution $(20 \mu \mathrm{l})$ was then added and the cells were incubated for $1 \mathrm{~h}$ to allow color development. The absorbance at $492 \mathrm{~nm}$ was measured as an index of the number of proliferating cells.

Statistical analysis. Results are presented as means \pm SD, and P-values were calculated by applying the two-tailed Student's $\mathrm{t}$-test to data from three independent experiments. Differences were considered statistically significant when $\mathrm{P}<0.05$.

\section{Results}

Autoacetylation at the K136 residue is conserved in ARDI variants. To investigate the autoacetylation activity of ARD1, three variants, GST-mARD1 ${ }^{225}$, GST-mARD1 ${ }^{235}$, and GST-hARD1 ${ }^{235}$, were purified and then subjected to an in vitro acetylation assay. As shown in Fig. 1A, GST-mARD1 ${ }^{225}$, GST-mARD1 ${ }^{235}$, and GST-hARD1 ${ }^{235}$ acetylated themselves in a time-dependent manner, whereas the control GST protein was not acetylated. To confirm the self-acetylation activity of ARD1, a dominant negative ARD1 was constructed with amino acid mutations at R82A and Y122F, blocking its binding to acetyl-CoA, and then subjected it to the in vitro acetylation reaction. Although wild-type $\mathrm{mARD} 1^{225}, \mathrm{mARD}^{235}$, and hARD $1^{235}$ acetylated themselves, the dominant negative ARD1 mutants were resistant to this acetylation, confirming that all ARD1 variants have self-activated autoacetylation activities (Fig. 1B).

The target site of autoacetylation was predicted using data from our previous study and sequence alignment (11). We have reported that hARD1 ${ }^{235}$ acetylation occurs at Lys136. Sequence alignment revealed that this site is conserved in $\mathrm{mARD}^{225}$ and $m A R D 1^{235}$ (Fig. 1C). To verify whether Lys136 is also a target site for the autoacetylation of $\mathrm{mARD} 1^{225}$ and $\mathrm{mARD} 1^{235}$, we constructed ARD1 mutants in which Lys136 was replaced 
A
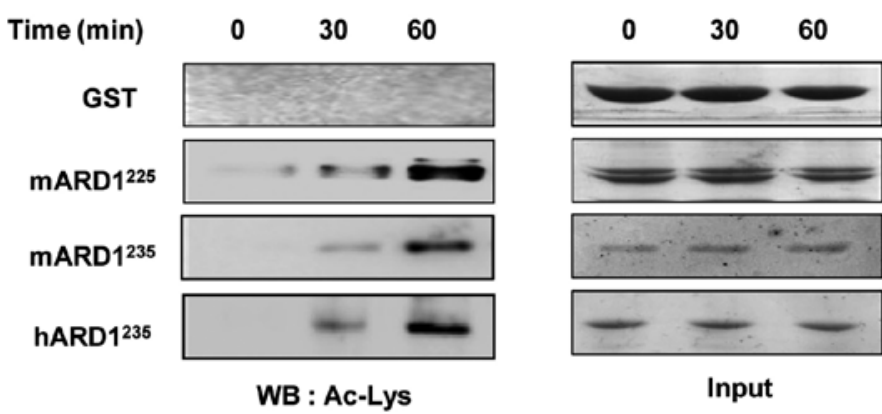

Input

B

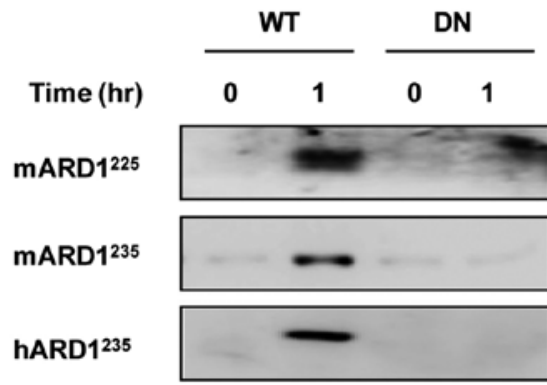

WB : Ac-Lys

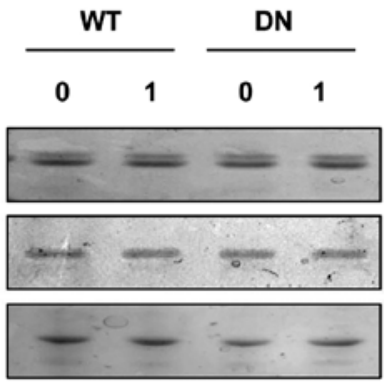

Input
C

mARD1225: LYSNTLNFQ

mARD1235: LYSNTLNFQ

hARD1235: LYSNTLNFQI
131

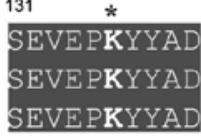

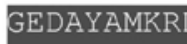

GEDAYAMKR

GEDAYAMKR
151

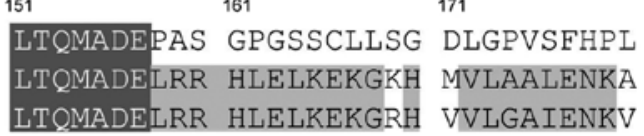

D
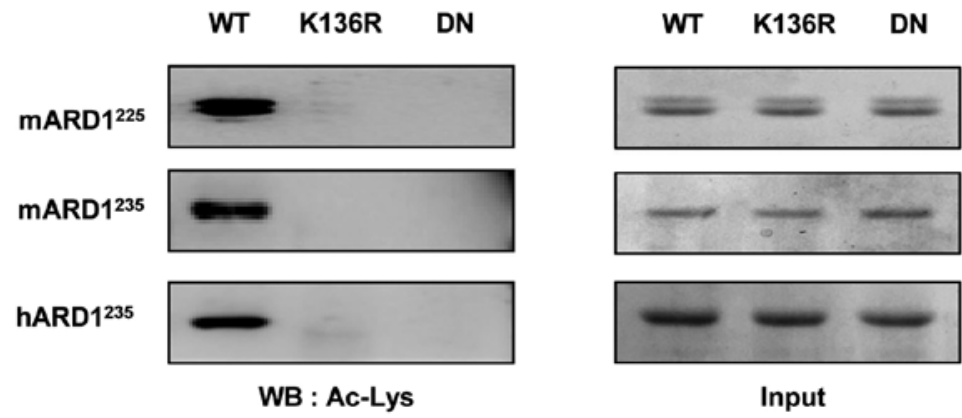

Figure 1. ARD1 variants self-acetylate the K136 residue in vitro. (A) Purified recombinants for GST-mARD1 ${ }^{225}$, GST-mARD1 ${ }^{235}$, and GST-hARD1 ${ }^{235}$ were subjected to the in vitro acetylation assay for 30 and $60 \mathrm{~min}$. Purified GST recombinant was subjected to the acetylation assay under the same conditions as a control. Acetylated proteins were detected by an anti-acetyl lysine (Ac-Lys) antibody. Total proteins were stained with Coomassie Brilliant Blue (input). (B) Wild-type GST-ARD1 recombinants (WT) and dominant negative GST-ARD1 (DN) with no catalytic activity were subjected to the in vitro acetylation assay. Acetylation status was analyzed with Ac-Lys antibody. Total proteins were stained with Coomassie Brilliant Blue (input). (C) The amino acid sequences of the ARD1 variants were compared. The conserved autoacetylation target site of ARD1 variants is indicated by an asterisk (*). (D) Purified GST recombinants of wild-type ARD1 (WT), K136R mutant ARD1 (K136R), and dominant negative ARD1 (DN) were subjected to the in vitro acetylation assay for $1 \mathrm{~h}$, followed by western blot analysis with Ac-Lys antibody. Total proteins were stained with Coomassie Brilliant Blue (input).

with Arg (K136R), and then performed the in vitro autoacetylation assay. As expected, K136R mutation abolished the autoacetylation activity of $\mathrm{mARD} 1^{225}$ and $\mathrm{mARD} 1^{235}$, as well as hARD1 ${ }^{235}$ (Fig. 1D). These results indicate that all ARD1 variants have autoacetylation activity and the target site is conserved at Lys136.
Autoacetylation of $m A R D 1^{225}$, but not $m A R D 1^{235}$ and $h A R D 1^{235}$, decreases HIF-la stability under hypoxic conditions. Autoacetylation is an important mechanism to regulate the enzymatic activity and the biological functions of acetyltransferase (17-20). Based on previous reports suggesting that ARD1 variants might have different biological functions 

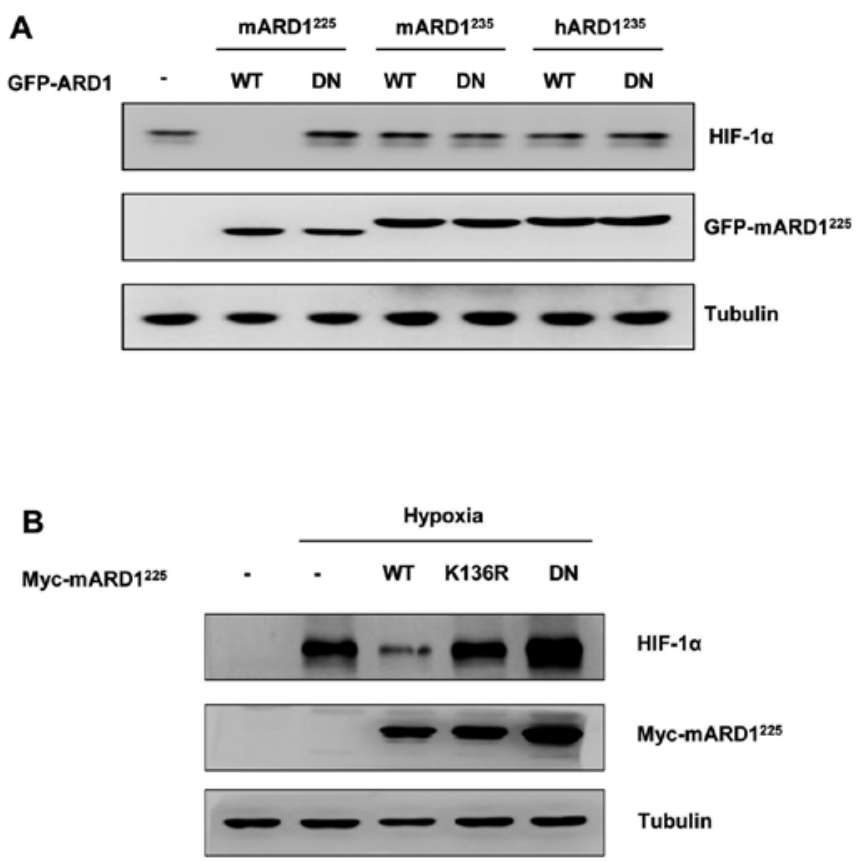

C
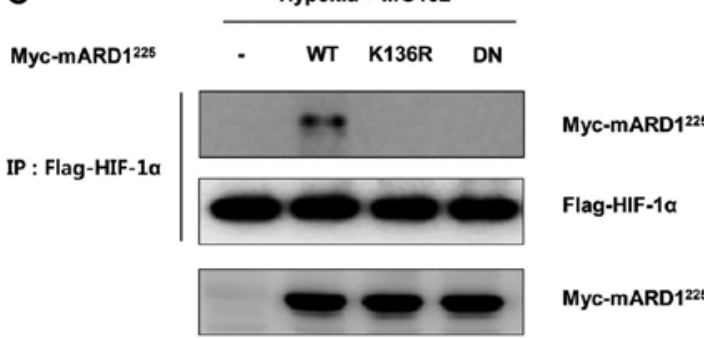

Figure 2. Autoacetylation of mARD $1^{225}$ decreases the stability of HIF-1 $\alpha$ protein. (A) HeLa cells were transfected with plasmids encoding GFP-tagged wild-type ARD1 (WT) or dominant negative ARD1 (DN), then incubated under hypoxic conditions for $4 \mathrm{~h}$. Total cell extracts were subjected to western blot analysis with anti-HIF-1 $\alpha$, anti-GFP, and anti-tubulin antibodies. (B) Myc-tagged plasmids for wild-type mARD1 ${ }^{225}$ (WT), K136R mutant mARD1 $^{225}$ (K136), and dominant negative mARD1 ${ }^{225}$ (DN) were transiently expressed in HeLa cells. HIF-1 $\alpha$ protein levels were analyzed by western blot analysis. (C) After transfection of HeLa cells with Flag-tagged HIF-1 $\alpha$ and Myc-tagged mARD1 ${ }^{225}$ plasmids, cells were treated with $10 \mu \mathrm{M}$ MG132 for $4 \mathrm{~h}$ under hypoxic conditions. Total cell lysates were immunoprecipitated with an anti-Flag antibody. The presence of Myc-mARD1 ${ }^{225}$ in the immunoprecipitates was examined with an anti-Myc antibody. Immunoprecipitated HIF-1 $\alpha$ protein and total cell extracts were analyzed with anti-HIF- $1 \alpha$ and anti-Myc antibodies, respectively.

(6), we hypothesized that even though ARD1 variants have common autoacetylation activity, this activity regulates each ARD1 variant separately. Thus, ARD1 variants have different biological functions depending on the specific isoform and physiological conditions.

Because it was reported that mARD1 ${ }^{225}$ decreases HIF-1 $\alpha$ stability by triggering protein degradation under hypoxic conditions $(2,21)$, we compared the effect of acetyltransferase activities of ARD1 variants on the stability of HIF-1 $\alpha$. HeLa cells were transfected with plasmids for wild-type or dominant negative ARD1 variants and incubated under hypoxic condi- tions. Consistent with the previous study, HIF-1 $\alpha$ protein was decreased in wild-type mARD1 ${ }^{225}$ transfected cells, but not in dominant negative mARD1 ${ }^{225}$ transfected cells (Fig. 2A). In addition, $\mathrm{mARD} 1^{235}$ and $\mathrm{hARD} 1^{235}$ did not change HIF-1 $\alpha$ protein levels regardless of whether wild-type or dominant negative mutants were used. These results not only confirm distinct functions of ARD1 variants, but also suggest a specific role of $\mathrm{mARD} 1^{225}$ in the regulation of HIF-1 $\alpha$ under hypoxic conditions.

To clarify the effect of mARD1 $1^{225}$ autoacetylation on the stability of HIF-1 $\alpha$, the K136R mutant mARD1 ${ }^{225}$ plasmid was transfected into HeLa cells and the HIF-1 $\alpha$ protein level was determined. As shown in Fig. 2B, wild-type mARD1 ${ }^{225}$ reduced HIF-1 $\alpha$ protein levels under hypoxic conditions while the K136R mutation inhibited the ability of $\mathrm{mARD} 1^{225}$ to decrease HIF-1 $\alpha$ protein levels. This indicates that $\mathrm{mARD}^{225}$ autoacetylation plays an indispensable role in the downregulation of HIF-1 $\alpha$ stability under hypoxic conditions. Interestingly, we also observed that neither the K136R mutant nor the dominant negative mARD1 ${ }^{225}$ could bind to HIF-1 $\alpha$, while the wild-type mARD1 ${ }^{225}$ binds to HIF-1 $\alpha$ under hypoxic conditions (Fig. 2C). These data suggest that mARD1 ${ }^{225}$ binds to HIF-1 $\alpha$ only after the acquisition of enzymatic activity through its autoacetylation.

Autoacetylation of $m A R D 1^{225}$ is required for $H I F-1 \alpha$ acetylation. When mARD1 ${ }^{225}$ regulates the stability of HIF-1 $\alpha$ under hypoxic conditions, the acetylation of the Lys532 residue in the ODD domain of HIF-1 is a critical step triggering HIF-1 $\alpha$ degradation $(2,21)$. Thus, we hypothesized that mARD1 ${ }^{225}$ autoacetylation stimulates the ability of $\mathrm{mARD} 1^{225}$ to acetylate the Lys532 residue in the ODD domain of HIF-1 $\alpha$. Because many studies have reported conflicting data on HIF-1 $\alpha$ acetylation in vitro $(2,22)$, we first determined whether mARD1 ${ }^{225}$ directly acetylated the Lys532 residue in the ODD domain in vitro. Purified recombinants for the His-tagged ODD domain in HIF-1 $\alpha$ and the GST-tagged mARD1 ${ }^{225}$ were prepared and subjected to acetylation in vitro. As shown in Fig. 3A, the ODD domain recombinant was successfully acetylated by the wild-type $\mathrm{mARD} 1^{225}$ recombinant, while the acetylation of the ODD domain of HIF-1 $\alpha$ was abrogated when the Lys532 residue was substituted with Arg (K532R). This demonstrated that mARD $1^{225}$ directly acetylates the Lys532 residue in HIF- $1 \alpha$.

To evaluate the effect of mARD1 ${ }^{225}$ autoacetylation on the acetylation of the ODD domain of HIF-1 $\alpha$, we subjected the $\mathrm{K} 136 \mathrm{R}$ mutant $\mathrm{mARD}{ }^{225}$ recombinant to the in vitro ODD domain acetylation assay. As expected, the K136R mutant mARD ${ }^{225}$ recombinant failed to acetylate the ODD domain of HIF-1 $\alpha$ in vitro, whereas the wild-type mARD $1^{225}$ recombinant successfully acetylated this domain (Fig. 3B). These results indicate that autoacetylation is the critical step to stimulate the catalytic activity of $\mathrm{mARD} 1^{225}$ that is required for the acetylation of the Lys532 residue in the ODD domain of HIF-1 $\alpha$.

Autoacetylation of $m A R D 1^{225}$ inhibits angiogenesis. When the HIF-1 $\alpha$ protein is stabilized under hypoxic conditions, it upregulates the expression level of several genes that promote angiogenesis (23-25). To determine the effect of mARD1 ${ }^{225}$ on hypoxia-induced angiogenic activity, we examined the 
A

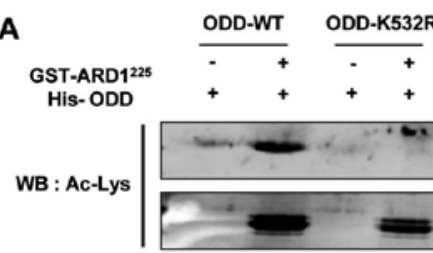

His-ODD-AC

GST-mARD1225-AC

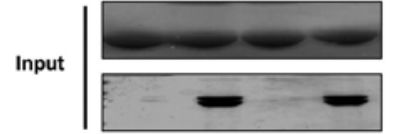

His-ODD

GST-mARD1225

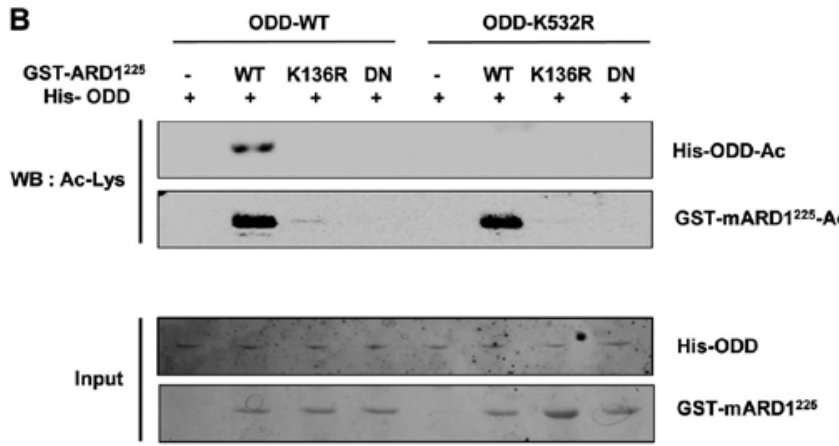

Figure 3. Autoacetylation of $\mathrm{mARD} 1^{225}$ is required for HIF-1 $\alpha$ acetylation. (A) Wild-type HIF-1 $\alpha$ ODD recombinant (ODD-WT) and K532R mutant HIF-1 $\alpha$ ODD recombinant (ODD-K532R) were purified and subjected to the in vitro acetylation assay for $1 \mathrm{~h}$ with or without $\mathrm{mARD}{ }^{225}$. Acetylation was analyzed by western blot analysis using an anti-Ac-Lys antibody. Total proteins were stained with Coomassie Brilliant Blue (input). (B) Purified GST-tagged recombinants of wild-type mARD1 ${ }^{225}$ (WT), K136R mutant $\mathrm{mARD1}^{225}$ (K136R), and dominant negative mARD1 ${ }^{225}(\mathrm{DN})$ were subjected to the in vitro acetylation assay with His-tagged ODD-WT or ODD-K532R recombinants. Acetylated proteins were detected using Ac-Lys antibody and total proteins were stained with Coomassie Brilliant Blue (input).

expression of $V E G F$ mRNA, a potent downstream target of HIF-1 $\alpha$ for promoting angiogenesis. Consistent with the data shown in Fig. 2B, wild-type mARD1 ${ }^{225}$ significantly decreased the mRNA level of VEGF. However, the K136R or dominant negative $\mathrm{mARD} 1^{225}$ had no influence on the expression of VEGF (Fig. 4A). In addition, conditioned media from cells transfected with wild-type mARD1 ${ }^{225}$ showed a strong inhibitory effect on endothelial tube formation, whereas conditioned media from cells transfected with K136R or dominant negative mARD $1^{225}$ had no effect on tube formation (Fig. 4B). These results indicate that the ability of $\mathrm{mARD} 1^{225}$ to inhibit tumor angiogenesis might be regulated by autoacetylation.

Autoacetylation of $m A R D 1^{235}$ and $h A R D 1^{235}$ but not $m A R D 1^{225}$ promotes cell proliferation. The distinct roles of autoacetylated ARD1 variants in regulating tumor growth were investigated under normoxic conditions. Because hARD1 ${ }^{235}$ promotes cell proliferation $(3,26)$, the effects of the ARD1 variants on cell growth were compared. Cell proliferation was analyzed after HeLa cells were transfected with plasmids for ARD1 variants. As shown in Fig. 5A and B, wild-type mARD1 ${ }^{235}$ and hARD1 ${ }^{235}$ significantly increased cell growth. However,
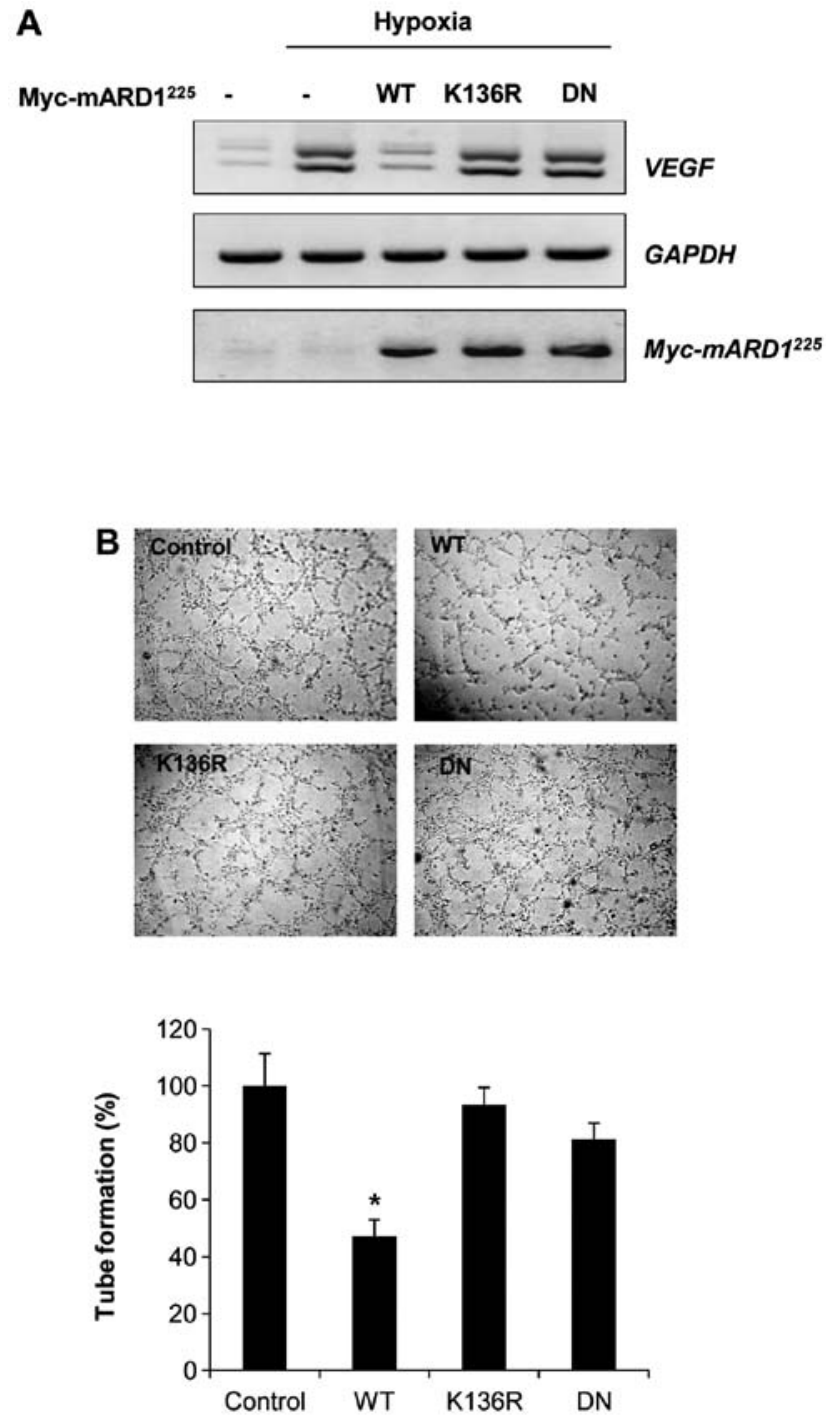

Figure 4. Autoacetylation of mARD1 ${ }^{225}$ inhibits angiogenesis. (A) HeLa cells were transfected with Myc-tagged mARD1 ${ }^{225}$ plasmids and incubated under hypoxic conditions for $24 \mathrm{~h}$. Total RNA was extracted for RT-PCR analysis. Expression of $V E G F$ and GAPDH mRNA was determined using specific primers. (B) HeLa cells expressing the mARD1 ${ }^{225}$ plasmids were incubated under hypoxic conditions for $24 \mathrm{~h}$ and conditioned media were collected. Human umbilical vein endothelial cells (HUVECs) were treated with conditioned media for $4 \mathrm{~h}$. Tube formation was photographed and quantified by counting the number of rings. ${ }^{*} \mathrm{P}<0.05$ versus control.

wild-type $m A R D 1^{225}$ did not alter cell growth, indicating distinct roles of ARD1 variants in the regulation of cell proliferation under normoxic conditions. Moreover, the abilities of mARD $1^{235}$ and hARD $1^{235}$ to enhance cell proliferation were abolished by K136R or dominant negative mutation of ARD1. This indicates that the autoacetylation activity of mARD1 $1^{235}$ and hARD1 ${ }^{235}$ is required for cell proliferation (Fig. 5B).

Based on our previous report showing that hARD1 ${ }^{235}$ induced cell proliferation is mediated by cyclin D1 (11), the effects of ARD1 variants on cyclin D1 levels were compared. After HeLa cells were transfected with plasmids for ARD1 variants, mRNA and protein expression levels of cyclin D1 were analyzed by RT-PCR and western blot analysis, respectively. Consistent with the data shown in Fig. 5A, mARD1 $1^{235}$ and $\mathrm{hARD} 1^{235}$ increased the expression level of cyclin D1. 

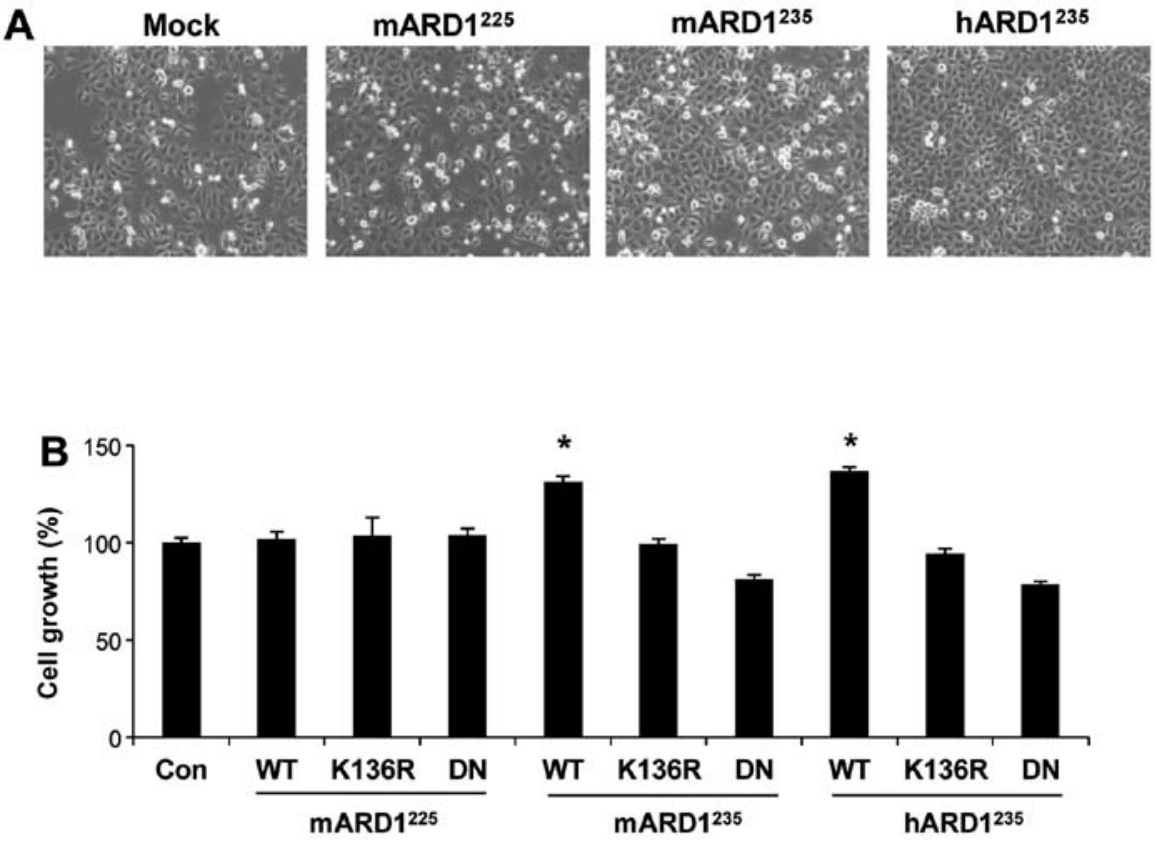

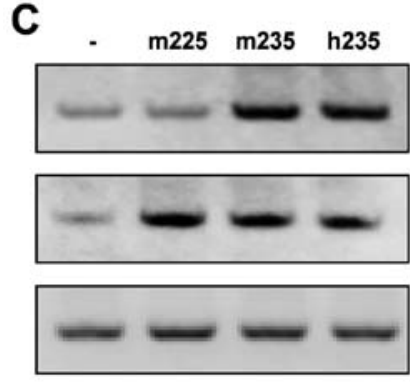

RT-PCR
D

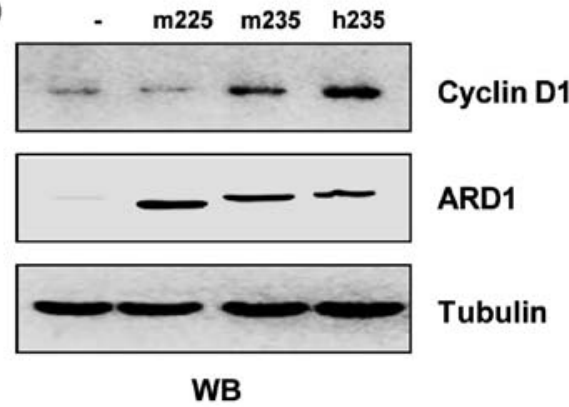

Figure 5. Autoacetylation of mARD1 ${ }^{235}$ and hARD1 $1^{235}$ but not mARD1 $1^{225}$ promotes cell proliferation under normoxic conditions. (A) HeLa cells were transfected with plasmids for ARD1 variants, then grown for 3 days and photographed. (B) After transfection of plasmids for wild-type and mutant ARD1 variants, cell growth was analyzed. "P $<0.05$ versus control. (C) HeLa cells were transfected with GFP-tagged mARD1 $1^{225}$, mARD1 ${ }^{235}$, and hARD1 ${ }^{235}$. The mRNA expression level of cyclin D1 was then analyzed by RT-PCR. (D) The expression level of cyclin D1 protein from HeLa cells expressing ARD1 variants was analyzed by western blot analysis using an anti-cyclin D1 antibody.

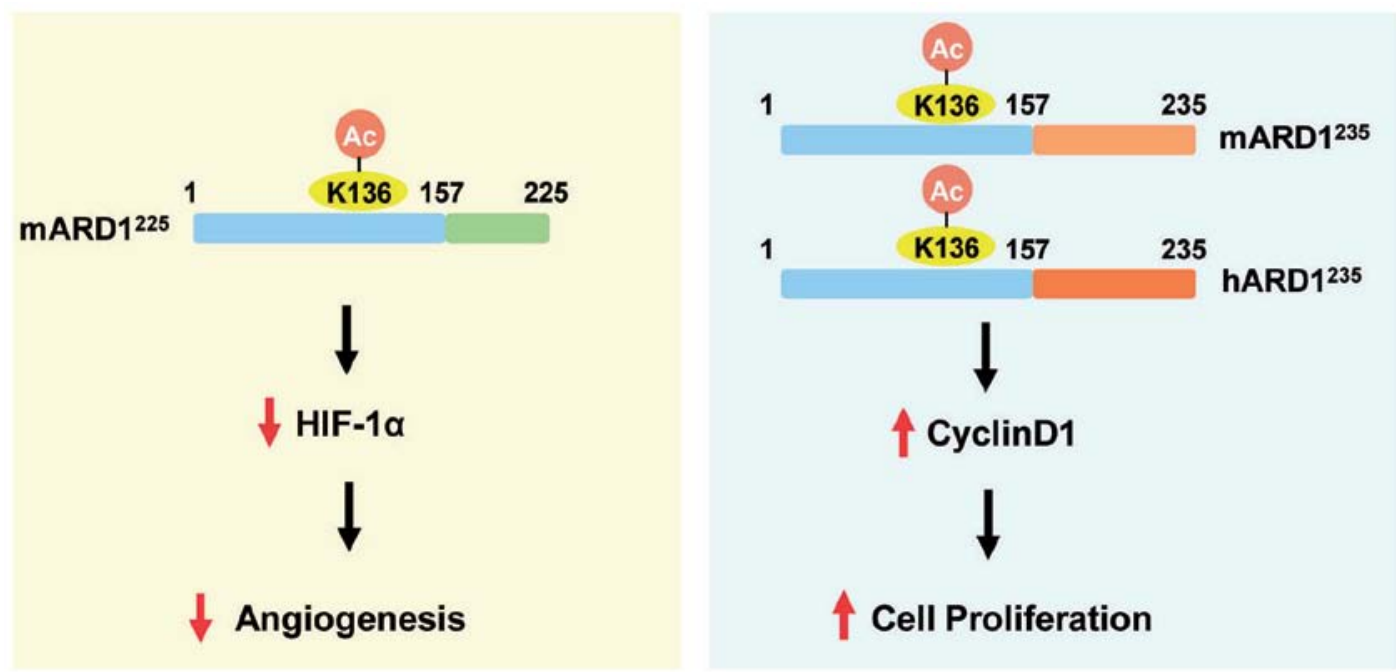

Figure 6. Isoform-specific roles of ARD1 autoacetylation in tumorigenesis. mARD1 ${ }^{225}$ autoacetylation inhibits angiogenesis through increased degradation of HIF-1 $\alpha$ under hypoxic conditions. Under normoxic conditions, autoacetylation of mARD ${ }^{235}$ and hARD1 ${ }^{235}$ promote cell proliferation by upregulating the expression of cyclin D1. Thus, autoacetylation of ARD1 variants differentially regulates angiogenesis and cell proliferation in an isoform-specific manner, depending upon the physiological condition. 
However, expression levels were unchanged by mARD1 ${ }^{225}$ (Fig. 5C and D). These results indicate that ARD1 variants have different effects on the expression of cyclin D1, demonstrating distinct functions of ARD1 variants in the regulation of cell proliferation under normoxic conditions.

\section{Discussion}

A number of acetyltransferases are known to be self-activated by autoacetylation (17-20). The present study provides data demonstrating that there is a conserved autoregulatory mechanism in ARD1 variants and shows how autoacetylation differentially regulates the enzymatic activities and biological functions of ARD1 variants, depending on the specific isoforms and physiological conditions.

We previously identified several ARD1 variants and suggested that they have distinct biological functions $(6,7)$. We also reported that hARD ${ }^{235}$ undergoes autoacetylation that enhances its cell proliferative activity (11). Because mARD1 ${ }^{225}$, mARD1 ${ }^{235}$, and hARD1 ${ }^{235}$ have a conserved acetyltransferase domain, we hypothesized that these three ARD1 variants have common autoacetylation activities. Consistent with this prediction, $\mathrm{mARD} 1^{225}, \mathrm{mARD}^{235}$, and $\mathrm{hARD} 1^{235}$ were observed to self-acetylate in vitro. In addition, the target site for autoacetylation was found to be conserved at the Lys136 residue.

Based upon differences in the amino acid sequences of the C-terminal region, the role of $\mathrm{mARD} 1^{225}$ could be different from that of $\mathrm{mARD}^{235}$ or hARD1 ${ }^{235}$. While the effects of hARD $1^{235}$ are related to cellular growth, mARD $1^{225}$ was originally found to inhibit angiogenesis (2). Thus, we speculated that, even though ARD1 variants share autoacetylation activity to acquire their acetylation activity, their biological functions might be distinct.

When mARD $1^{225}$ modulates angiogenesis under hypoxic conditions, it directly interacts with and acetylates the Lys532 residue in the ODD domain of HIF-1 $\alpha$, triggering degradation of the HIF-1 $\alpha$ protein (2). The significance of mARD1 ${ }^{225}$ autoacetylation was clearly revealed by our observation that the K136R mutant mARD1 ${ }^{225}$ could not acetylate the Lys532 residue in the ODD domain of HIF-1 $\alpha$ in vitro, whereas wild-type mARD1 ${ }^{225}$ acetylated it. Accordingly, stability of the HIF-1 $\alpha$ protein was reduced in wild-type mARD1 $1^{225}$ expressing cells, but not in K136R mutant mARD1-expressing cells. Furthermore, blocking autoacetylation diminished the ability of $\mathrm{mARD} 1^{225}$ to inhibit VEGF expression and endothelial tube formation. From these results, we conclude that autoacetylation serves as a key switch for regulating the antiangiogenic function of mARD $1^{225}$ under hypoxic conditions.

In contrast to autoacetylation of $\mathrm{mARD}{ }^{225}$, the autoacetylation of $\mathrm{mARD} 1^{235}$ and $\mathrm{hARD} 1^{235}$ had no effect on angiogenesis. Under hypoxic conditions, the stability of the HIF-1 $\alpha$ protein was unchanged by either wild-type or mutant $\mathrm{mARD} 1^{235}$ and hARD1 ${ }^{235}$. However, autoacetylation of $\mathrm{mARD} 1^{235}$ and $\mathrm{hARD} 1^{235}$ played an important role in cell growth under normoxic conditions. Consistent with our previous report (11), cell proliferation was remarkably increased by wild-type mARD $1^{235}$ and hARD $1^{235}$, but not by K136R mutants. However, in terms of cell growth, autoacetylation of $\mathrm{mARD} 1^{225}$ appeared unrelated to cell proliferation under normoxic conditions. Neither wild-type nor the K136R mutant of mARD $1^{225}$ had any effect on cell growth. Cyclin D1 was increased by mARD1 ${ }^{235}$ and hARD1 ${ }^{235}$, but not by mARD1 ${ }^{225}$. These data support our previous suggestion about distinct roles of ARD1 variants in tumor angiogenesis and cell growth. In addition, data from the present study also suggest that the distinct role of ARD1 variants is selectively regulated by autoacetylation in an isoform-specific manner (Fig. 6).

Alternative splicing is a widespread process generating multiple transcripts from a single mRNA precursor. This process commonly occurs during gene expression and contributes to protein diversity (27). Indeed, more than half of all mammalian genes are alternatively spliced, and diverse transcripts produced from alternative splicing often have distinct functions $(28,29)$. Alternative splicing of exon 8 of mouse ARD1 leads to the production of discrete ARD1 isoforms ( $\mathrm{mARD} 1^{225}$ and $\mathrm{mARD} 1^{235}$ ) that have distinct functions in tumorigenesis (7). Different subcellular localizations of $\mathrm{mARD} 1^{225}$ and $\mathrm{mARD} 1^{235}$ may correlate with their distinct functions (7). In contrast to mice, alternative splicing of ARD1 exon 8 does not occur in humans. Thus, only hARD1 ${ }^{235}$ is present in humans, indicating that alternative splicing of ARD1 is a species-specific event. To understand the evolutionary events leading to species-specific ARD1 isoforms, it might be necessary not only to identify diverse ARD1 variants in other species such as rat, rabbit, and monkey but also to define the detailed individual functions of ARD1 variants in each species.

In conclusion, the present study reveals different roles of ARD1 variants in angiogenesis and cell proliferation. ARD1 variants use a common regulatory system called autoacetylation to regulate their individual roles. Although autoacetylation is a conserved mechanism that ARD1 variants use to regulate their enzymatic activities, depending on physiological conditions, autoacetylation selectively regulates the biological functions of ARD1 in an isoform-specific manner. These findings offer new insight into the distinct functions of ARD1 isoforms in cancer development, and provide a clue as to how ARD1 variants could be selectively targeted in cancer treatment.

\section{Acknowledgements}

We thank Dr Gregg L. Semenza for providing the Flag-tagged HIF-1 $\alpha$ plasmid. This study was supported by the Global Research Laboratory Program (2011-0021874), Global Core Research Center (GCRC) Program (2011-0030001), National Research Foundation (NRF) grant (2013-036038) funded by the Ministry of Science, ICT, and Future Planning (MSIP) and Basic Science Research Program through the NRF of Korea funded by the Ministry of Education (2013R1A1A2058956).

\section{References}

1. Driessen HP, de Jong WW, Tesser GI and Bloemendal H: The mechanism of N-terminal acetylation of proteins. CRC Crit Rev Biochem 18: 281-325, 1985.

2. Jeong JW, Bae MK, Ahn MY, et al: Regulation and destabilization of HIF-1alpha by ARD1-mediated acetylation. Cell 111: 709-720, 2002.

3. Lim JH, Park JW and Chun YS: Human arrest defective 1 acetylates and activates beta-catenin, promoting lung cancer cell proliferation. Cancer Res 66: 10677-10682, 2006. 
4. Ohkawa N, Sugisaki S, Tokunaga E, et al: N-acetyltransferase ARD1-NAT1 regulates neuronal dendritic development. Genes Cells 13: 1171-1183, 2008.

5. Shin DH, Chun YS, Lee KH, Shin HW and Park JW: Arrest defective-1 controls tumor cell behavior by acetylating myosin light chain kinase. PLoS One 4: e7451, 2009.

6. Kim SH, Park JA, Kim JH, et al: Characterization of ARD1 variants in mammalian cells. Biochem Biophys Res Commun 340: 422-427, 2006.

7. Chun KH, Cho SJ, Choi JS, Kim SH, Kim KW and Lee SK Differential regulation of splicing, localization and stability of mammalian ARD1235 and ARD1225 isoforms. Biochem Biophys Res Commun 353: 18-25, 2007.

8. Arnesen T, Kong X, Evjenth R, et al: Interaction between HIF-1 alpha (ODD) and hARD1 does not induce acetylation and destabilization of HIF-1 alpha. FEBS Lett 579: 6428-6432, 2005.

9. Fisher TS, Etages SD, Hayes L, Crimin K and Li B: Analysis of ARD1 function in hypoxia response using retroviral RNA interference. J Biol Chem 280: 17749-17757, 2005.

10. Bilton R, Mazure N, Trottier E, et al: Arrest-defective-1 protein, an acetyltransferase, does not alter stability of hypoxia-inducible factor (HIF)-1alpha and is not induced by hypoxia or HIF. J Biol Chem 280: 31132-31140, 2005.

11. Seo JH, Cha JH, Park JH, et al: Arrest defective 1 autoacetylation is a critical step in its ability to stimulate cancer cell proliferation. Cancer Res 70: 4422-4432, 2010.

12. Arnesen T, Gromyko D, Pendino F, Ryningen A, Varhaug JE and Lillehaug JR: Induction of apoptosis in human cells by RNAi-mediated knockdown of hARD1 and NATH, components of the protein $\mathrm{N}$-alpha-acetyltransferase complex. Oncogene 25: 4350-4360, 2006.

13. Ren T, Jiang B, Jin G, et al: Generation of novel monoclonal antibodies and their application for detecting ARD1 expression in colorectal cancer. Cancer Lett 264: 83-92, 2008.

14. Shim JH, Chung YH, Kim JA, et al: Clinical implications of arrest-defective protein 1 expression in hepatocellular carcinoma: a novel predictor of microvascular invasion. Dig Dis 30: 603-608, 2012.

15. Wang ZH, Gong JL, Yu M, et al: Up-regulation of human arrestdefective 1 protein is correlated with metastatic phenotype and poor prognosis in breast cancer. Asian Pac J Cancer Prev 12: 1973-1977, 2011.
16. Yu M, Gong J, Ma M, et al: Immunohistochemical analysis of human arrest-defective-1 expressed in cancers in vivo. Oncol Rep 21: 909-915, 2009.

17. Thompson PR, Wang D, Wang L, et al: Regulation of the p300 HAT domain via a novel activation loop. Nat Struct Mol Biol 11: 308-315, 2004.

18. Santos-Rosa H, Valls E, Kouzarides T and Martinez-Balbas M: Mechanisms of P/CAF auto-acetylation. Nucleic Acids Res 31: 4285-4292, 2003.

19. Blanco-Garcia N, Asensio-Juan E, de la Cruz X and MartinezBalbas MA: Autoacetylation regulates $\mathrm{P} / \mathrm{CAF}$ nuclear localization. J Biol Chem 284: 1343-1352, 2009.

20. Stavropoulos P, Nagy V, Blobel G and Hoelz A: Molecular basis for the autoregulation of the protein acetyl transferase Rtt109. Proc Natl Acad Sci USA 105: 12236-12241, 2008.

21. Lee MN, Lee SN, Kim SH, et al: Roles of arrest-defective protein 1(225) and hypoxia-inducible factor 1alpha in tumor growth and metastasis. J Natl Cancer Inst 102: 426-442, 2010.

22. Murray-Rust TA, Oldham NJ, Hewitson KS and Schofield CJ: Purified recombinant hARD1 does not catalyse acetylation of Lys532 of HIF-1alpha fragments in vitro. FEBS Lett 580: 1911-1918, 2006.

23. Semenza GL: Targeting HIF-1 for cancer therapy. Nat Rev Cancer 3: 721-732, 2003.

24. Hong SS, Lee H and Kim KW: HIF-1alpha: a valid therapeutic target for tumor therapy. Cancer Res Treat 36: 343-353, 2004.

25. Lee JW, Bae SH, Jeong JW, Kim SH and Kim KW: Hypoxiainducible factor (HIF-1)alpha: its protein stability and biological functions. Exp Mol Med 36: 1-12, 2004

26. Arnesen T, Thompson PR, Varhaug JE and Lillehaug JR: The protein acetyltransferase ARD1: a novel cancer drug target? Curr Cancer Drug Targets 8: 545-553, 2008.

27. Nilsen TW and Graveley BR: Expansion of the eukaryotic proteome by alternative splicing. Nature 463: 457-463, 2010.

28. Brett D, Pospisil H, Valcarcel J, Reich J and Bork P: Alternative splicing and genome complexity. Nat Genet 30: 29-30, 2002.

29. Mironov AA, Fickett JW and Gelfand MS: Frequent alternative splicing of human genes. Genome Res 9: 1288-1293, 1999. 